\title{
Mixed Ductal-Neuroendocrine Carcinoma of the Pancreas
}

National Cancer Institute

\section{Source}

National Cancer Institute. Mixed Ductal-Neuroendocrine Carcinoma of the Pancreas. NCI

Thesaurus. Code C6879.

A carcinoma that arises from the pancreas showing a mixture of ductal and

neuroendocrine malignant cells in both the primary tumor and in the metastatic sites. 